\begin{tabular}{|c|c|}
\hline \multirow{3}{*}{ 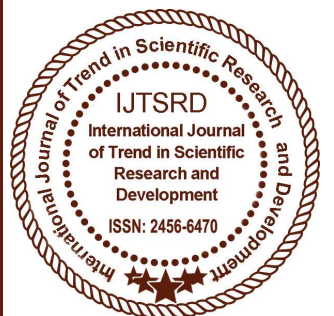 } & $\begin{array}{l}\text { International Journal of Trend in Scientific } \\
\text { Research and Development (IJTSRD) }\end{array}$ \\
\hline & International Open Access Journal \\
\hline & ISSN No: 2456 - 6470 | www.ijtsrd.com | Volume - 2 | Issue - 2 \\
\hline
\end{tabular}

\title{
Study on Real-time Operating System and its Scheduling Procedures
}

\author{
Durgesh Raghuvanshi \\ B. Tech, Department of Computer Science \\ IILM Academy of Higher Learning, Greater Noida, Uttar Pradesh, India
}

\section{ABSTRACT}

This paper describes current research in real time operating system and its scheduling procedures. The scheduling procedure is the backbone of any operating systems. In this paper we analyses the application of scheduling theory to dependable real time systems are reviewed with a brief summary and conclude that different types of scheduling procedures are used in real time operating system. Issues covered include static and dynamic algorithms, application of real time operating system, rate monotonic and deadline monotonic with examples. This paper describes about characteristics of real time operating system.

Keywords: RTOS- Real time operating system, $\boldsymbol{R M S}$ Rate monotonic scheduling, DM- Deadline monotonic, $\mathbf{E D F}$ - Earliest deadline first, $\mathbf{L S F}$ - Least stack time first

\section{Introduction}

Real time (computing, communication \& information) systems have become increasingly important in everyday life. A real time system is required to complete its work and deliver its services a timely basis. Many real time systems are embedded in sensors and actuators and functional digital controllers.

In these types of applications, signals arrive periodically at fixed periods. When the signals arrive they must be classified as hard real time or soft real time. The report then reviews the literature on priority driven scheduling algorithms which can be divided into dynamic and fixed priority. In this paper the choice of algorithm can influence the behavior of a real-time system. Many real time systems are embedded in sensors and actuators and function as digital controllers. With the help of this paper I will attempt provide an overview of many of the different available real time procedures. This paper also covers the industrial sector and marketing sector.

\section{Real Time Operating System}

The concept of deadline hard and soft real time system were introduced above throughout this section definitions are given of the terms commonly used in scheduling literature.

Real time in operating system is the ability of the operating system to provide a required level of service in a bounded response time.

\subsection{Soft RTOS}

- In a soft real time system, it is considered undesirable, but not catastrophic if deadlines are occasionally missed.

- Also known as "best effort" systems.

- Most modern operating system can serve as the base for short real time systems.

For example:

1. Multimedia transmission and reception.

2. Website and services.

3. Computer games 
International Journal of Trend in Scientific Research and Development (IJTSRD) ISSN: 2456-6470

\subsection{Hard RTOS}

- A hard real time system has time critical deadlines that must be met; otherwise a catastrophic system failure can occur.

- Requires formal verification/ guarantees of being to always meet its hard deadlines (except for fatal errors).

For example:

1. Air traffic control

2. Vehicle subsystems control

3. Nuclear power plant control

\section{Scheduling in RTOS}

More information about the tasks is known as:

- Number of tasks

- Resources requirements

- Execution time

- Deadlines

\subsection{Scheduling algorithms in RTOS}

- CLOCK DRIVEN

1. All parameters about jobs known in advance.

2. Schedule can be computed offline or at some regular time instances.

3. Minimum runtime overhead.

4. Not suitable for many applications.

\section{- WEIGHTED ROUND ROBIN}

1. Jobs scheduled in FIFO manner.

2. Time quantum given to jobs in proportional to its weight.

- For example:

1. High speed switching network.

2. QOS guarantee.

\section{Priority SchedulingGreedy/list/ Event Driven}

- Processor never left idle when there are ready tasks.

- Processor allocated to processes according to priorities.
1. Static - at design time

2. Dynamic -at runtime

\subsection{Priority Scheduling}

- Earliest Deadline First (EDF).

Process with earliest deadline given highest priority

- Least Stack Time (LSF).

slack $=$ relative-execution left

1. Forperiodic task.

2. Tasks priority is inversely proportional to its period.

\section{SCHEDULING PROCEDURES}

\subsection{Static Scheduling}

Static priority based algorithms are relatively simple to implement but lack flexibility. They are arguably the most common in practice and have a fairly complete theory. They work well at fixed periodic tasks but do not handle a periodic tasks particularly well although there are some methods.

Static priority based scheduling algorithms have two disadvantages, which have received a significant amount of study. There low processor utilization and poor handling of a periodic and soft- deadline tasks have prompted researchers to search for ways to combat these deficiencies. When all the scheduling decisions are made prior to the running of the system then it is static and offline.

Rate Monotonic Scheduling is optimal meaning that if any static priority scheduling algorithm can meet all deadlines. The method of assigning the priorities to the set of processes assigning the priorities as a monotonic function of the rate of process. Rate monotonic scheduling provides simple inequality comparing total processor utilization to a theoretically determined bound that serves as a sufficient condition to ensure that all processes will complete their work by the end of their periods.

$$
\sum_{i=1}^{n} \frac{C i}{T i} \leq n\left(2^{\frac{1}{n}}-1\right)
$$

Where, $C i=$ the execution time, $T i=$ period associated with periodic task. 


\begin{tabular}{|l|l|l|}
\hline Process & $\begin{array}{l}\text { Execution } \\
\text { time }\end{array}$ & Period \\
\hline P1 & 1 & 8 \\
\hline P2 & 2 & 5 \\
\hline P3 & 2 & 10 \\
\hline
\end{tabular}

The utilization will be

$$
\frac{1}{8}+\frac{2}{5}+\frac{2}{10}=0.725
$$

The sufficient condition for 3 process under which we conclude that the system is schedulable is

$$
U=3\left(2^{\frac{1}{3}}-1\right)=0.77976 \ldots
$$

Since,

$$
0.725<0.77976 \ldots
$$

The system is surely schedulable.

Deadline Monotonic is a scheduling algorithm is an algorithm that uses fixed priority preemptive according to the deadline of the given process. With deadline monotonic priority assignment tasks are assigned priorities according to their deadlines; the task with the shortest deadline being assigned the highest priority.

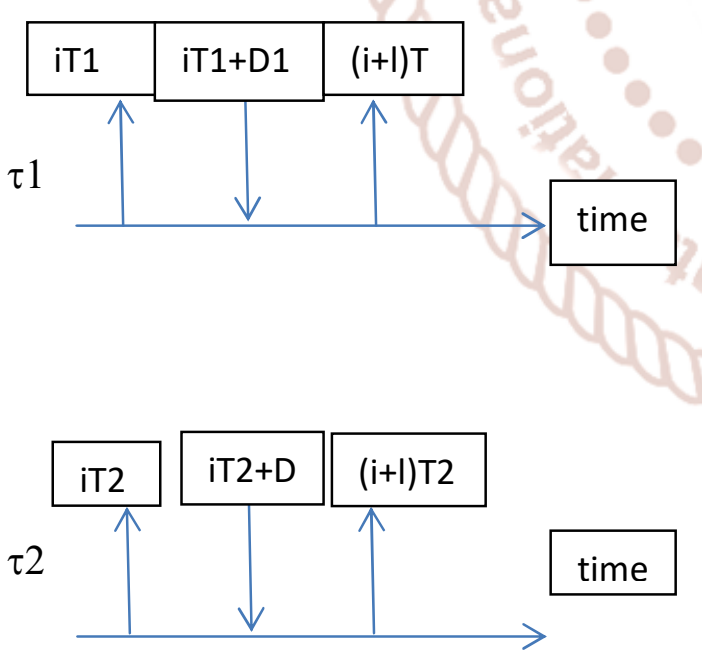

Since T1 has the highest priority it claims the processor whenever it has an outstanding computational requirement. This will occur for the first $\mathrm{C} 1$ units of each period $\mathrm{T} 1$.

\subsection{Dynamic Scheduling}

Dynamic Scheduling procedures are those procedures in which the priorities to the processes are given to them at the time of execution of the task. The main motto to this is adapt to dynamically changing progress of process. The periodic tasks are real time which are activated irregularly at some unknown and possible unbounded rate.

$$
\sum_{i=1}^{n} \frac{C i}{P i} \leq 1
$$

\section{CONCLUSION}

Real Time Operating System have been the heroes in most of the technological areas, right from fuel injection system to nuclear reactor control, satellite control, global positioning systems, and fully equipper hi tech warfare aircrafts. And the best is yet to come! Development and maintenance of complex systems require that researchers develop tools for automatic system integration, monitoring, scheduling, verification and testing and reconfiguration.

\section{REFERENCE}

1. A.Burns and A.J.Welling's real-time systems and their scheduling procedures.

2. ChenyangLu, CSE 467S Embedded Computing Systems.

3. Kaushik Ghoshand Anjana Tudu,109CS0196 schedulability analysis of periodic task of uniprocessor system on real time system.

4. P.A. Laplantte, real time system design and analysis An engineer hand book IEEE Press, 1993.

5. Real time operating system Wikipedia.org/wiki/real time operating system. 